\title{
SUR LA BIFURCATION DU DIBOTHRIOCEPHALUS LATUS
}

\author{
Par N. LEON
}

Cette anomalie, observée chez un certain nombre de téniadés, a été signalée par divers auteurs. Toutefois, en ce qui concerne le Dibothriocephalus latus, je ne connais qu'un seul cas de bifurcation à plusieurs segments ; c'est le cas de Bremser (1819) que les auteurs semblent vouloir ignorer. R. Blanchard (1894), lorsqu'il expose ses idées sur la " bifurcation de la chaîne " (p. 31), dit que l'on n'a pas encore observé de bifurcation chez le Dibothriocephalus latus : " elle est pour ainsi dire encore ignorée chez les parasites de l'homme ". Il ne dit pas un seul mot du cas de Bremser. Max Braun (1901), parlant de cette anomalie (p. 1618), dit qu'elle a été observée par Albini sur un exemplaire de Bothriocephalus latus et que ladite bifurcation ne comportait que deux segments. Lui non plus ne dit rien du cas de Bremser.

Quoi qu'il en soit, je ne relève dans aucun de ces cas le désir d'expliquer les causes qui produisent cette malformation chez le Dibothriocephalus latus. Nous présentons, dans le présent article, deux cas de bifurcation chez ce cestode. Ces cas nous ont paru dignes d'une description spéciale, étant donné que, dans un cas, la chaîne compte treize anneaux et que, dans l'autre cas, elle en compte dix.

Dans le premier cas, il s'agit d'un Dibothriocephalus latus (fig. 1) qui mesure 80 centimètres de longueur. La tête manque, ainsi que la série des anneaux qui la continuent jusqu'aux anneaux mûrs. Les anneaux ont tous leur aspect normal. On ne remarque, à la surface, aucun indice d'érosion ou d'altération quelconque. Seule, la partie terminale du ver présente sur vingt centimètres environ les anomalies suivantes : tout d'abord, une fénestration centrale qui s'étend sur trois anneaux, après quoi viennent 14 anneaux réguliers, entiers ; là, une seconde fénestration centrale apparaît qui s'étend sur quatre anneaux, à la suite desquels se succèdent neuf anneaux d'aspect normal ; après le neuvième anneau, on aperçoit une troisième fénestration qui, elle aussi, s'étend sur quatre anneaux, lesquels sont à leur tour suivis de deux anneaux réguliers. C'est après ces deux anneaux réguliers qu'a lieu la bifurcation en deux chaînes : celle de gauche, comportant huit anneaux et celle de droite, qui est constituée par dix anneaux.

Annales de Parasitologie, $\mathrm{T}$. IV, $\mathrm{N}^{\circ} 3 .-1^{\text {er }}$ juillet 1926 , p. 236-240. 
Le second cas nous offre un Dibothriocephale latus (fig. 1) muni de sa tête et mesurant 2 mètres, 15 de long. Les segments présen-

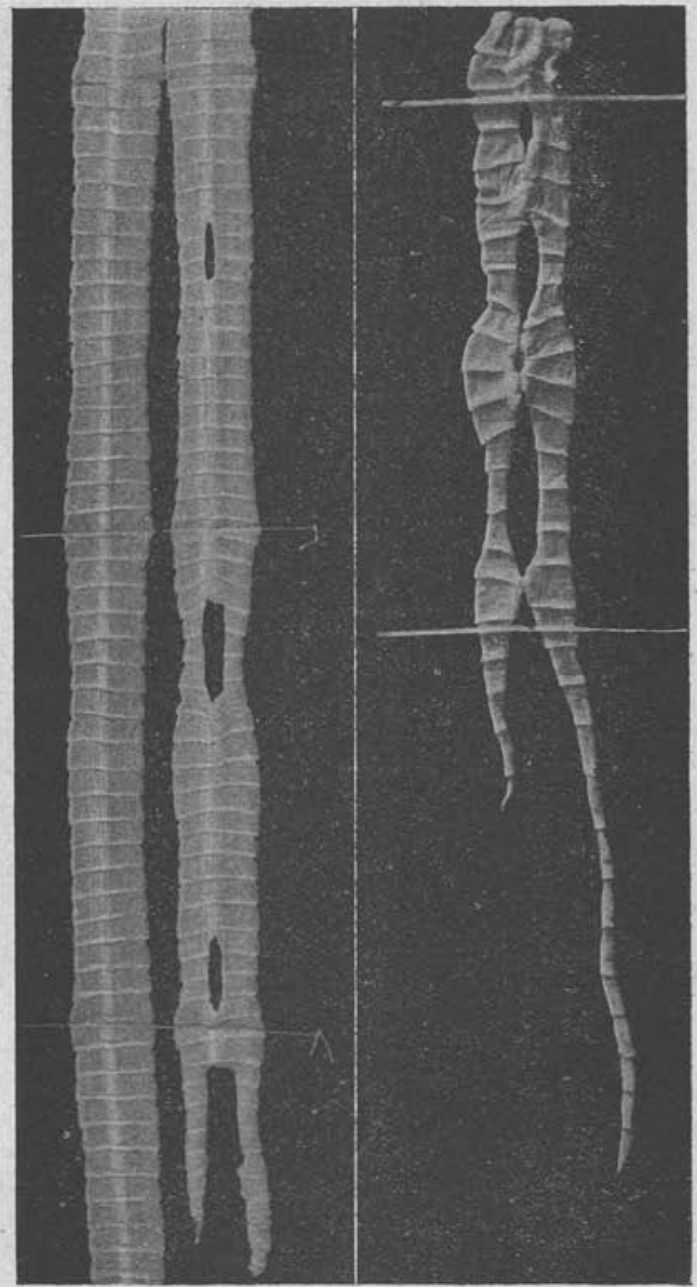

Fig. 1. - Fenestration et bifurcation de la chaîne chez Dibothriocephalus latus. A gauche, premier cas; à, droite, second cas.

tent tous un aspect normal, sauf la partie finale de la chaîne, mesurant 15 centimètres, où l'on relève les anomalies suivantes : quatre fénestrations; la première s'étend sur deux anneaux; la 
deuxième s'étend sur trois anneaux ; la troisième, qui est la plus petite, s'étend sur deux anneaux et la quatrième, la plus grande, s'étend sur six anneaux. Après la quatrième perforation, on voit une double chaîne de proglottis ; la chaine gauche est. plus courte et est constituée par six anneaux ; quant à la chaîne droite, qui est plus longue, elle est formée de treize anneaux. Chaque anneau.de cette double chaîne est d'un tiers moins large que les anneaux d'aspect normal. Les anneaux des deux bifurcations, tant celle de droite que celle de gauche, ne présentent aucune trace d'altération des éléments histologiques. Les bords internes des anneaux qui regardent vers la ligne médiane du strobile, où se trouvaient situés les organes de reproduction, sont réguliers, nets et limités par la cuticule.

En ce qui concerne les causes qui produisent la bifurcation de la chaîne, les auteurs ne sont pas d'accord. Les uns croient que la bifurcation s'effectue après la fénestration et à cause de celle-ci ; les autres pensent que la bifurcation est due à l'intercalation d'un anneau qui se répète plusieurs fois à la même place, si bien qu'au lieu de produire un seul anneau intercalé, il produit une série d'un certain nombre d'anneaux. Si l'on s'en rapporte aux exemplaires que nous reproduisons dans la figure 1, on doit admettre la première hypothèse. Par suite du peu de largeur des anneaux chez le $D$. latus, les perforations s'étendent en longueur suivant l'axe du ver, et intéressent plusieurs anneaux. Or, quand l'anneau terminal vient à être perforé, les deux moitiés du strobile s'écartent l'une de l'autre en formant une bifurcation à droite et une bifurcation à gauche.

L'origine de la bifurcation de la chaine remonte à l'origine de la perforation de cette chaine. Avant d'expliquer l'origine des perforations, nous constatons que l'on distingue chez le Dibothriocephalus latus deux sortes de perforations :

$1^{\circ}$ Les perforations centrales ou fenestrées, qui occupent le centre des anneaux, en réduisant l'anneau à un simple eadre, comme dans la figure 1.

$2^{\circ}$ Les perforations intercalaires, comme dans la figure 2. Ces perforations intercalaires n'ont pas du tout les mêmes causes que les perforations centrales. A notre avis, la cause de ces perforations est l'affaiblissement de la cuticule et des fibres musculaires longitudinales, qui, en se brisant, font que les anneaux se séparent les uns des autres. Ces sortes de perforations sont beaucoup plus rares que les perforations centrales et n'ont aucun rapport avec la bifurcation de la chaîne. 
Il y a, au sujet des causes qui produisent les perforations centrales, un certain nombre d'avis que nous avons résumés dans une note ayant pour titre : "Sur la fenestration du Bothriocephalus latus ", note qui a paru dans le Zoologischer Anzeiger en 1907. Nous avons également confirmé alors l'hypothèse de Bremser, en nous basant sur les études histologiques de deux bothriocéphales fenestrés; nous avons démontré que les perforations étaient produites par l'utérus. Lorsque l'utérus s'enfle exagérément sous la

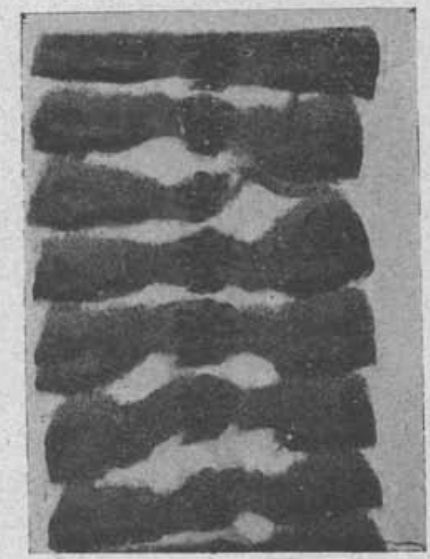

Fig. 2. - Perforations intercalaires chez le Dibothriocephalus latus.

pression des œufs qui s'y entassent, la cuticule du dessus de l'utérus se tend de plus en plus et, finalement, se déchire. Les sucs digestifs s'accumulant, détruisent progressivement, tout d'abord l'utérus, ensuite la couche corticale qui se trouve sur le côté opposé, puis la cuticule et en dernier lieu les parties avoisinantes, jusqu'à ce qu'enfin se produise la perforation complète de l'anneau.

Blanc (1888) attribue la cause première des perforations " à un développement irrégulier, anormal de la cuticule de quelques anneaux chez certains vers, constituant une sorte d'état pathologique, complété par une action digestive externe, mais qui disparaîtra au bout d'un certain temps et eela pendant la croissance des anneaux et de la chaîne ". Cette hypothèse ne peut se soutenir, par le fait qu'il n'arrive jamais que l'enveloppe tégumentaire s'amincisse davantage autre part que sur la ligne médiane des proglottis au-dessus de la rosette utérine. 
Les deux exemplaires que nous présentons ici (fig. 1) prouvent une fois de plus que la fénestration est la cause de la bifurcation de la chaîne chez le D. latus et que cette fenestration est due à une ponte excessive qui a pour effet la rupture des parois utérines. $\mathrm{Si}$, comme le suppose Danysz (1888), la fenestration avait pour cause les petits dépôts graisseux, la rupture devrait se produire encore sur d'autres points, sur les bords par exemple, et non pas seulement sur le milieu des anneaux, c'est-à-dire là où se trouve la rosette de l'utérus.

Chez les autres cestodes, chez lesquels l'utérus et les organes reproducteurs sont situés sur les côtés de chaque anneau, les fenestrations, ressemblant à des entailles, se produisent sur les côtés des anneaux, toujours exactement au même endroit, comme nous l'avons observé chez le Dipylidium (1909). Chez les ténias qui. ont l'utérus situé sur la ligne médiane des segments les fenestrations se produisent toujours sur la ligne médiane.

\section{RÉSUMÉ}

Je signale dans cette note deux cas de bifurcation de la chaîne chez le Dibothriocephalus latus. Dans un de ces cas, l'une des chaînes (fig. 1) est constituée par treize anneaux. On peut, sur ces deux exemplaires, observer que la bifurcation s'effectue en arrière des fénestrations centrales, qui sont provoquées par une ponte excessive, ayant pour effet la rupture des parois de l'utérus.

\section{BIBLIOGRAPHIE}

Blanc (H.). - Trnia saginata et Bothriocephalus latus avec anneaux perforés. Lausanne, imprimerie Corbaz et $\mathrm{C}^{\text {ie }}, 1888$.

Blanchard (R.). - Sur quelques cestodes monstrueux. Progrès Nédical, 1894.

Braun (M.). - Bronn's Klassen und Ordnungen des Tierreichs, Vermes. Abtheilung I. b. Cestodes, 1901.

Buemser. - Uber lebende Würmer im lebenden Menschen. Wien, Carl Schaumburg et $\mathrm{C}^{i}, 1819$.

Danysz (J.). - Recherches sur un ténia fenêtré. Journal de l'anatomie, XXIV, avec une planche, 1888 .

Leon (N.). - Sur la fenestration du Bothriocephalus lalus. Zoologischer Anzeiger, XXXII, n॰ 8, 1907.

- Uber eine Missbildung von Dipylidium caninum. Zoologischer Anzeiger, XXXIV, $\mathrm{n}^{\circ}$ 5, 1909.

Laboraloire de Parasilologie de la Faculté de médecine de Jassy (Roumanie). 